\title{
LA TENSIÓN ENTRE LAS PRIORIDADES DEL ESTADO Y LOS INTERESES DEL MERCADO
}

\author{
Sergio A. Berumen \\ Real Academia de Ciencias Morales y Políticas \\ Madrid, España \\ lee_berumen@racmyp.es
}

\begin{abstract}
Resumen
En la realidad social, el Estado y el mercado realizan funciones de coordinación específicas; estos agentes están estrechamente relacionados, pero entre ellos hay permanente tensión. El presente artículo es una reflexión acerca de la tensión que se genera entre las prioridades del Estado y los intereses del mercado. Las bases teóricas desde las cuales se parte corresponden a un enfoque netamente liberal, si bien se atiende diversos matices que resultan muy convenientes (por ejemplo, liberalismo neoschumpeteriano, liberalismo austriaco y liberalismo de la elección pública). El interés, lejos de proponer una visión concluyente, es mostrar una parte del amplio abanico de posibilidades conceptuales liberales desde las cuales se puede analizar este objeto de estudio.
\end{abstract}

Palabras clave: prioridades del Estado, intereses del mercado, pensamiento liberal.

\begin{abstract}
In the social reality, the State and the market carryout specific coordination functions; these agents are closely related but their relation is permanently tense. This article is a reflection on the tension generated between the priorities of the State and the market interests. The theoretical base from which it parts is basically liberal, although it has several minor changes which result pretty convenient (v.g. neoschumpeterian liberalism, Austrian liberalism, and public choice liberalism). The objective, far from proposing a concluding vision, is to demonstrate a part of the wide spread of conceptual liberal possibilities from which this object of study can be analyzed.
\end{abstract}

Key words: State priorities, market interests, liberal thought. 


\section{Introducción}

La sociedad actual está formada por una serie de agentes que permiten la convivencia social, tales como el Estado y el mercado. La conjunción de estos agentes da lugar a un macrosistema político, económico y social que constantemente hace sentir su influencia en la toma de decisiones sobre el desarrollo y el funcionamiento de las instituciones. De este modo, las instituciones están configuradas por una pluralidad de características históricas, naturales, culturales, sociales, económicas y políticas. En este contexto, el desarrollo de una sociedad está condicionado por las características que definen la división del trabajo y las relaciones entre los grupos sociales que perviven dentro de los agentes y las instituciones que los forman.

La creación de instituciones tiene el propósito de lograr medios eficaces que promuevan el orden y el cumplimiento de la legalidad en la sociedad. Sin embargo, si bien estos agentes se encuentran estrechamente relacionados, su vínculo es de constante tensión. El presente estudio se centra en la tensión dinámica que se genera a partir de la interacción entre los intereses públicos y los intereses particulares, tanto entre la sociedad y los individuos como entre los estados y los mercados, entre la política y la economía, entre la riqueza y el poder, y entre el poder y la política. En este complejo proceso político-económico existe una participación multilateral de estados nacionales y mercados (nacionales e internacionales).

La actuación de estos agentes se consuma a través de la asignación de recursos y la satisfacción de las necesidades de los participantes. Mayormente, esto corresponde a una división del trabajo eficiente.
La contrapartida de la división del trabajo son los costos de coordinación, los cuales definen en gran medida la eficiencia alcanzada. Tales costos se generan a través de las interacciones y la participación de las instituciones en la división del trabajo y afectan tanto a instituciones públicas como privadas. Los diferentes sistemas políticos, económicos y sociales que surgen de la configuración entre el Estado y el mercado son alentados o combatidos dependiendo de los costos de administración que generan y los diferentes grados de eficiencia que alcanzan en la asignación de recursos.

El presente artículo es una reflexión cuyo interés es convertirse en tema de discusión y debate desde diferentes perspectivas analíticas. El cuerpo teórico a partir del cual se ha desarrollado corresponde a un enfoque liberal, si bien atiende diversos matices que nos han parecido necesarios (por ejemplo, liberalismo neoschumpeteriano, liberalismo austriaco y liberalismo de la elección pública). De este modo, el interés, más allá de proponer una visión concluyente para el análisis de la tensión entre las prioridades del Estado y los intereses de mercado, es mostrar una parte del amplio abanico de posibilidades conceptuales liberales desde las cuales se puede analizar este objeto de estudio.

\section{La convivencia entre el Estado y el mercado}

En un sistema de economía abierta, los participantes del mercado toman sus propias decisiones y están obligados a asumir las consecuencias de tales decisiones. En el sistema capitalista de talante liberal el Estado desempeña un papel de garante y valedor en torno a: a) la protección de la propiedad privada y la libertad económica 
a través de la creación de marcos legales, b) la promoción y la protección de los intereses de los individuos y las empresas privadas, c) el respeto a la legalidad, d) el cumplimiento de las reglas creadas para el fomento de la libre y leal competencia y e) el respeto al equilibrio logrado por consumidores y oferentes sobre los niveles de producción y precios (Rodríguez Braun, 2005: 47).

La influencia que ha desempeñado el Estado sobre el mercado y sus participantes a lo largo de la historia se ha expresado en un conjunto de aspectos y características: valores, intereses, cultura y dimensión institucional del Estado y el mercado, entre otros. En este contexto, el Estado hace uso del poder político para decidir «quiénes pueden obtener qué y cómo» en una sociedad. El qué se refiere a los recursos económicos, pero también a los derechos y obligaciones, y el cómo se refiere a los aspectos jurídicos que dan certidumbre a los actos y al cumplimiento de los contratos. Por tanto, la definición del papel del Estado en la economía y su participación en el ámbito económico y social están determinados por la forma en que las estructuras de poder han definido la división del trabajo entre sus distintos niveles institucionales y de acuerdo con las consecuencias ponderadas que se esperan de esa división del trabajo.

El control que ejerce el Estado sobre los aspectos estratégicos de la economía se evidencia, con frecuencia, a través de una responsabilidad pública cuyos propósitos son: a) el respeto a la legalidad, b) lo que se entienda por equidad social, c) lo que se entienda por ejercicio del libre comercio, d) la defensa de la soberanía nacional, e) el sentimiento de protección a los sectores más desprotegidos de la población, entre otros.
Para Fuà y Materazzi (2004), estos aspectos se encuadran en lo que consideran la sociedad civil, la cual pervive en un escenario hostil, semejante a un campo de batalla, donde predomina el interés privado e individual; lo que conduce a una lucha de todos contra todos en la que cada uno es para sí su único fin y lo demás se vuelve irrelevante. Este enfoque neomarxista, que encuentra sus hondas raíces en el pensamiento de Marx y Engels, se refiere a la búsqueda de la extinción de la división de clases y supone que sólo entonces será posible que las funciones políticas históricas del Estado se transformen en funciones administrativas cuyo fin último sea la persecución y el mantenimiento de los intereses de la mayoría.

Desde esta perspectiva, se deduce que el sistema subsiste en una permanente pugna entre intereses privados, lo que origina una acumulación desigual de la riqueza y una división del trabajo que eventualmente puede ser perjudicial para determinados sectores sociales, razón por la cual se presume que el Estado tiene la obligación y la responsabilidad de evitar que se creen extremas desigualdades en la distribución de la riqueza y, a partir de ello, grandes diferencias sociales.

Otros enfoques menos radicales justifican la participación del Estado en el mercado cuando el segundo se muestra incapaz o manifiesta tendencias a una equívoca actuación, y principalmente se refieren a las distorsiones o desequilibrios que genera la «mano invisible» de Adam Smith (Salvon y Ska, 2002: 35). La intervención en el desarrollo de la cosa pública (res publicae) se justifica con el argumento de que el Estado no debe permanecer impasible ante el libre juego del mercado, porque la tendencia natural de este se orientará a 
favorecer sólo los intereses de unos cuantos. Por tanto, la presuntamente diligente «mano visible» del Estado se encargará de, inclusive, sacrificar el bien particular si la promesa es alcanzar un bien general mayor. La intervención de esa "mano visible» del Estado en el mercado se observa a través de la imposición (o eliminación) de tarifas, aranceles e impuestos, la emisión de dinero, la creación de subvenciones, el establecimiento de permisos previos, los límites a la producción y la connivencia entre políticos y empresarios y la concesión de derechos y privilegios (por ejemplo, la típica tendencia a gobernar por decreto, como la alegre decisión de aumentar el salario mínimo o las pensiones -cosa que es, casualmente, recurrente en fechas previas a la celebración de elecciones), entre otros.

En todo caso, lo que no alberga dudas es que las políticas impuestas por el Estado inciden en la división del trabajo y, por consiguiente, en la generación de los costos de transacción, lo cual determinará la eficiencia y la eficacia económica en la asignación de recursos de las distintas instituciones y del país. La actuación del Estado en la gestión de la cosa pública genera costos de transacción, derivados de los efectos de sus relaciones con el mercado (además de los que tienen que ver con su estricta responsabilidad, como el hacer valer la preeminencia de la ley y el mantenimiento del orden público).

En lo concerniente al diseño de la política económica, mediante el control de precios el Estado combate la inflación, fomenta la inversión e incentiva o modera el crecimiento. Estas decisiones implican consecuencias en el mercado como, por ejemplo, la descapitalización de algunas empresas (mientras que otras se pueden ver beneficiadas), el desvío de recursos hacia la producción de bienes y servicios, el combate contra la piratería y, en general, la economía subterránea. En el caso particular de la definición de la presión fiscal, Santibáñez y Puigcerdá (2005: 18-21) han estudiado la forma en la cual los impuestos se utilizan como mecanismos para desalentar el consumo y la producción, y en calidad de catalizadores que alteran el volumen de intercambio y, por tanto, impactan -usualmente de manera negativa- en el equilibrio del mercado.

Pero la cuestión se complica aun más cuando el Estado interviene en el quehacer productivo (y, por tanto, en el mercado); este proceder usualmente trae consigo el incremento (tanto en el número como en el tamaño) de las instituciones gubernamentales y de los servidores públicos que en ellas trabajan. Esta situación ha implicado severas distorsiones en la división del trabajo, porque un amplio porcentaje de la generación de empleo corre por cuenta del propio Estado. Se trata de un gran contingente de funcionarios públicos, los que a su vez necesitan una enorme cantidad de funcionarios que los dirijan, los supervisen $\mathrm{y}$ los vigilen, todo lo cual suma un numeroso ejército de burócratas, fortalecidos en gremios que son capaces de ejercer mucha presión y de impedir la implementación de una correcta política económica de reducción del gasto.

La gremialización de la burocracia ha tendido a la formación de grandes sindicatos, que en repetidas ocasiones han ejercido una coacción tal que al final el Estado ha terminado por otorgar concesiones sui géneris que inclusive pueden contravenir la ley. El gigantismo del Estado ha ocasionado que sus funciones se tornen más torpes y, por añadidura, que sea más fácilmente infiltrado por la corrupción y el cohecho, de los que, 
desde luego, el capital privado es víctima y coprotagonista. Este trastornado desempeño de las funciones del Estado genera por lo menos tres graves problemas: a) la corrupción, cuyos elevados costos, por su naturaleza, son difíciles de contabilizar, b) el errático y manipulador proceder de algunos funcionarios (agrupados en sindicatos), que, por la presión de la que son capaces de ejercer, genera una incertidumbre jurídica, traducida en una inhibición de la inversión privada y c) el incremento en los estándares del riesgo-país.

Finalmente, la influencia del Estado en el mercado se evidencia cuando crea, adquiere o protege empresas a las que les otorga concesiones extraordinarias (por ejemplo, exoneración del pago de impuestos, cesión de terrenos y ayudas públicas, y la abierta protección de sectores productivos y de sus empresas, como es el caso de los «campeones nacionales») con el interés de incentivar la creación de polos de desarrollo o concentración industrial. En estos casos la discusión surge entre si el apoyo a las empresas alienta el desarrollo de las localidades o si, en contraposición, tales respaldos generan una competencia desleal para las empresas que ya están instaladas en otros lugares, dentro de los mismos países $\mathrm{o}$, inclusive, en terceros países.

Este dilema se puede afrontar desde diversos puntos de vista; aquí exponemos dos. Para el enfoque liberal neoschumpeteriano, es imprescindible la alianza entre el Estado y las empresas, mediante la cual el primero está obligado a conceder todos los medios necesarios para que los sectores productivos sean más competitivos. Para el enfoque liberal austriaco, en cambio, está claro que la «sabiduría» del mercado en modo alguno debe ser intervenida por regulación alguna de parte del Estado.
A nuestro modo de ver, tan dañina es la agobiante y excesiva regulación como la ausencia total de ella.

\section{La tensión entre las prioridades del Estado y los intereses del mercado}

Hasta el siglo XIX, la división de poderes entre el Estado y el mercado no fue clara (en muchos casos ambos poderes coincidían en una misma persona o institución). En la actualidad, el Estado y el mercado son dos agentes con leyes propias y con funciones específicas dentro de la sociedad; no obstante, con frecuencia se siguen entremezclando sus funciones e intereses, lo cual induce a imbricaciones entre las competencias y las obligaciones de ambos. Desde la creación misma del Estado moderno (que data del Renacimiento italiano, entre los siglos XV y XVI), la división del poder político y del poder económico ha generado una frecuente tensión, cuando no un abierto conflicto (Pérez Prendes y Muñoz de Arraco, 1998: 227).

Las tensiones entre las prioridades del Estado y los intereses del mercado se originan, principalmente, en lo referente a la asignación y la distribución de la riqueza y el poder. Por un lado, los mercados se encargan de la asignación y la distribución de los recursos escasos, mientras que el Estado se responsabiliza de la asignación y la distribución del poder. Por tanto, el Estado y el mercado reflejan los intereses de quienes los integran. Aquí se establece que tras la distinción entre la esfera económica y la esfera política reaparece la distinción entre la singulorum utilitas y el status rei publicae.

Existe una dicotomía pública-privada entre la sociedad del citoyen (ciudadano), 
que se preocupa por el interés colectivo y la del bourgeois (burgués), que contempla el beneficio de los intereses privados (Bobbio, 1989: 28-45). Por tanto, el Estado es el conjunto de acciones colectivas, mientras que el mercado es el conjunto de acciones individuales. Bajo la óptica del enfoque tradicional, la supremacía del Estado sobre el mercado está justificada por la contraposición del interés colectivo y el interés privado, lo que hace necesaria la subordinación o la supresión del interés privado.

Asimismo, los estados se ocupan de los intereses y los valores sociales a través de sus acciones colectivas, como el cumplimiento del derecho al voto, la reglamentación de la representación social y la configuración de las instituciones de la nación. Los intereses generales se ven manifestados e interpretados por el Estado mediante mecanismos políticos (como los procesos de elección popular). Sin embargo, es difícil saber cuánta preferencia social existe detrás de una determinada acción del Estado. La eficiencia de la representación del interés social en las acciones del Estado estará en función de la naturaleza de las instituciones gubernamentales y de las características de elección y representación popular.

Por su parte, el mercado pone en marcha un mecanismo de acción que está motivado por intereses individuales y condicionado por aspectos como la competencia y el comportamiento individual. Los participantes del mercado se conducen de acuerdo con sus intereses personales. De ahí que el Estado en numerosas ocasiones haya justificado su intervención en el mercado al establecer que este no considera los intereses públicos y los sacrifica en aras de intereses privados. Según este planteamiento, se presume que el mercado no tiene convicciones sociales y no se preocupa por la justicia ni la igualdad.
A partir de lo anterior, el Estado y el mercado se encuentran relacionados a través de los sistemas de producción, intercambio y distribución. La relación que existe entre ambos agentes es de carácter dinámico; es decir, se transforma a lo largo del tiempo. El Estado influye en el mercado y viceversa, dando lugar a un constante intercambio y contraposición de intereses: en la realidad social causas económicas producen consecuencias políticas, y causas políticas generan consecuencias económicas. Por ende, los cambios en la dinámica del mercado y del Estado afectan directamente la asignación y la distribución de la riqueza $\mathrm{y}$ el poder.

\subsection{La interacción dinámica entre los agentes}

En este escenario es necesario estudiar la tensión dinámica que se genera a partir de la interacción entre: a) los intereses públicos y los intereses particulares, b) la sociedad y los individuos, c) los estados y los mercados, d) la política y la economía, e) la riqueza y el poder y f) el poder y la política. En este complejo proceso político-económico se produce una participación multilateral de estados nacionales e instituciones nacionales e internacionales. En suma, es una red dinámica e interdependiente de relaciones entre estados y mercados, entre el poder y la riqueza.

Dentro de la vasta complejidad de competencias, es conveniente identificar la eficiencia que se logra a través de la división del trabajo dentro de las instituciones en la sociedad, y la configuración económica, política y social de la nación y el progreso económico y social. La división del trabajo y sus consecuencias sobre la eficiencia de una economía están determinadas por la forma como un país haya definido esa 
división del trabajo entre las diferentes categorías institucionales. Por tanto, la eficiencia no solo debe darse en el campo de la asignación de recursos, sino también desde una perspectiva dirigida a la satisfacción de las necesidades y deseos de la sociedad (García Maynes, 1994: 134).

La otra cara de la división del trabajo entre las instituciones y los procesos son los costos que se pueden originar. Estos costos determinan los sistemas de relaciones entre las instituciones que participan en la división del trabajo y definen, en gran medida, la eficiencia económica y política de la sociedad. A partir de lo anteriormente señalado, el Estado y el mercado legitiman su actuación y su grado de intervención en la sociedad de acuerdo con el grado de eficiencia y la mayor maximización del bienestar al menor costo.

En este contexto, el mercado tiene la función de coordinar los sistemas productivos y de consumo y de generar beneficios para ambos. Mientras que el mercado desempeña su labor de coordinación a través de mecanismos como el precio, el Estado aporta un conjunto de instrumentos que son complementarios al mercado y las empresas; el correcto y diligente funcionamiento de ellos genera certidumbre y estabilidad en los mercados y, su ausencia, su parcialidad, su torpeza o su ineficiencia generan incertidumbre, la cual se traduce en elementos que incrementan el riesgo.

Desde un punto de vista analítico, el Estado y el mercado trabajan en conjunto en la producción y distribución de los bienes y servicios que la sociedad requiere. Pero, más allá del debate entre liberales y progresistas, el Estado es responsable de la seguridad, el orden público, la educación cívica, así como de garantizar el cumplimiento de los contratos y la protección de las patentes y los derechos de autor, entre muchos otros aspectos; principalmente se interesa en que sus objetivos generales prevalezcan sobre los objetivos económicos, y que los intereses generales prevalezcan sobre los individuales o de grupo (Roncagliolo, 2003: 16-18).

\subsection{Las fallas del Estado y las fallas del mercado}

El debate sostenido entre las prioridades del Estado y los intereses del mercado se desarrolla en torno a las fallas de ambas entidades. Cuadrado Roura (2005: 82-96) ha identificado algunas de las fallas del mercado más emblemáticas: a) la existencia y preeminencia de mercados no competitivos, b) la existencia de factores externos que subsisten al margen o contra la ley, c) los resultados deficientes en cuanto a generación de empleo, crecimiento, desarrollo y estabilidad económica, d) la existencia de rendimientos a escala crecientes y e) la insuficiente o insatisfactoria distribución de la renta. Generalmente, la competencia imperfecta genera ineficiencias y, por ende, una menor satisfacción de los consumidores; en un escenario de mercados imperfectos donde participan pocos productores, estos pueden llegar a ejercer poder sobre los precios.

Por su parte, el mercado acusa los excesos del Estado y promueve la no-intervención basándose en las fallas del sector público, tales como: a) las ineficiencias en la producción de bienes públicos y sociales, b) las dificultades en el control o la gestión de los monopolios que explotan los recursos naturales, c) la existencia de externalidades generadas en el sector público, d) la ignorancia de los costos reales con los que opera el sector público, e) la inseguridad pública y 
f) la corrupción y el cohecho. Con frecuencia, en las empresas públicas los intereses de los burócratas ejercen influencia en la determinación de precios. Hay la creencia de que cuando cada una de las esferas, Estado y mercado, se desempeña correctamente, la respuesta de las otras instituciones hacia ellas tenderá a ser eficiente. Esto conduce a afirmar que cuando una determinada institución lleva a cabo sus actividades de forma eficiente; es decir, maximizando el beneficio, no se justifica la intervención de otra institución en su desempeño.

La cuestión que se deriva de estas fallas es la definición de los objetivos estatales y privados, los cuales pueden llegar a ser ambiguos y generar controversias cuando los estados y los mercados tienen metas similares pero sus actividades son motivadas por intereses y valores diferentes. Es frecuente que la reacción del Estado a problemas particulares sea distinta a la reacción del mercado. En el aspecto psicosocial, el mercado persigue la eficiencia y exige la configuración de un sistema que lo promueva, mientras que el Estado busca el cumplimiento de valores como la imparcialidad, la justicia y la equidad. En esta situación el Estado justifica su intervención con el argumento del logro y el mantenimiento de los objetivos de carácter social: justo en este contexto reside la tensión entre la eficiencia y la equidad.

La visión yuxtapuesta tiende a evaluar si es posible: a) repartir sin crear, b) repartir más de lo que se es capaz de crear, c) repartir menos de lo que se es capaz de crear o d) inhibirse de repartir y confiar en que el mercado se encargará de hacerlo de la mejor manera posible. El problema es que los gobiernos muchas veces olvidan que para crear, antes es preciso lograr la asignación eficiente de los recursos, la preeminencia de una sana competitividad y el incremento de la productividad, lo que en términos sociales se traduce en que todos los individuos que forman la sociedad tengan la posibilidad de mejorar sus condiciones de vida, lo cual no necesariamente implica una lucha montaraz por la igualdad. Para Amatto (2004: 27-31), una sociedad que luche por la igualdad será, invariablemente, generadora de pobreza, mientras que una sociedad que abogue por la competitividad generará riqueza. En realidad, esta es una expresión típica de las ideas liberales expresadas hace medio siglo por la Escuela Austriaca, cuyos viejos destellos aportan nuevas luces para la solución de problemas recientes.

\subsection{Enfoques teóricos relevantes para el análisis}

Desde la perspectiva del Public Choice (Elección Pública, la cual se ha orientado prioritariamente a estudiar la actividad pública de los gobiernos $\mathrm{y}$, en particular, los costos que esta causa), es factible analizar las fallas y beneficios del Estado y contrastarlos con las fallas y beneficios del mercado (Buchanan y Tullock, 1962; Buchanan, 1969). Este enfoque subraya las funciones sociales ineludibles y necesarias del Estado, pero cuestiona el desinteresado y altruista desempeño de la administración pública (Buchanan, 1992). El Public Choice señala que los grupos de poder en el seno de los gobiernos (y que forman el denso entramado económico, político y social) suelen anteponer sus intereses personales, inclusive por encima del interés común (lo anterior va más allá de la superación de las fallas que se mencionaron anteriormente). A partir de este hecho, el origen de toda política económica diseñada por los gobiernos no se rige, entonces, por el máximo bienestar social; es decir, la superación de esas fallas, sino por el logro 
de objetivos personales, de partido o gremiales.

Por consiguiente, las ideas defendidas por el Public Choice establecen que las presuntas bienintencionadas acciones de los políticos generalmente se preocupan más de la distribución que de la producción, porque estos, en el afán de tratar de satisfacer el interés general, creen erróneamente que los recursos son más abundantes de lo que en realidad son (Buchanan, 1985). Pero el afán regulatorio es aun más letal, debido a que en su búsqueda irracional de controlar el máximo posible, el Estado se ve obligado a crecer más allá de lo necesario y de lo administrativamente funcional, hasta llegar al extremo de que secuestra el interés público, cuando en realidad lo que prevalece es el interés privado de políticos, sindicatos y demás gremios interesados en que continúe el statu quo (Buchanan, 1975).

Este afán regulatorio se alimenta de la creencia de que en la relación contractual establecida entre el pueblo y el Estado, el segundo es el responsable de proporcionar los servicios públicos, lo cual conduce a que sea fácilmente manipulable lo que se entiende por servicio público. Esta relación contractual ha sido generalmente aceptada en prácticamente todos los países, pero no por aceptada es correcta. Este modelo ha sido presa de los intereses de burócratas que lo defienden bajo el argumento de que el Estado es el mejor vigilante y protector de la vida, la libertad y la propiedad de las personas.

Empero, en la práctica estos nobles valores han languidecido y en su lugar han imperado los de los políticos maximizadores de votos, que se valen de campañas de propaganda frecuentemente alimentadas de intimidación y chantaje. De esta manera, el recurso de la promesa ha sido utilizado sin importar los costos e, incluso, sabiendo de antemano que no se cumplirá a cabalidad con lo ofrecido. Autores como Smitz y Zubizarreta (2003: 42-43) han demostrado que los presupuestos de los gobiernos son, como promedio, el doble y en algunos casos hasta el quíntuplo de lo que deberían ser bajo el rigor de una administración eficiente. En la creencia de que tamaño significa poder, la burocracia se ha apropiado de la idea del interés público.

Lo anterior se complementa con lo dicho en su día por Joseph A. Schumpeter (1942) en cuanto a que los gobiernos tienden a perseguir objetivos que promuevan y justifiquen su permanencia en el poder. El hecho de que los individuos que buscan participar en la esfera política están alentados por una combinación de razones públicas y privadas los motivará a buscar servir a la sociedad y servirse a sí mismos. Tanto el Estado como el mercado están constituidos por individuos que tomarán sus decisiones y llevarán a cabo sus elecciones sobre la base de su interés personal (ergo, los servidores públicos tratarán de beneficiar su interés privado a través de sus acciones públicas), independientemente de que ello implique, o no, la superación de las fallas.

Los planteamientos de Schumpeter y del Public Choice, entre otros trabajos, fueron considerados por el Nobel de Economía de 1991, Ronald Coase (1963 y 1999), cuando escribió sus artículos más destacados (el primero en 1937 y el segundo en 1960), en los cuales señaló que cualquier sistema de asignación de precios tiene un costo imprescindible de determinar. Empero, es un problema muy generalizado el que sea prácticamente imponderable especificar cuánto cuesta mantener al Estado y su gestión. 
En muchos países en desarrollo ha sido una constante que los gobiernos mantengan en permanente formulación cuánto cuesta mantener al Estado, pero la realidad es que tales reflexiones, solo en casos muy específicos, han conducido a una reducción del volumen del sector gobierno. A la luz de los hechos, se ha demostrado que los temores de los progresistas -desde los neomarxistas hasta los neoestructuralistas-, que vaticinaban una debacle si los estados reducían aun más sus competencias y ámbitos de gestión, eran totalmente infundados (Vuscovic, 1975: 53). En realidad, los procesos de desregulación y privatización vividos en la práctica por la totalidad de los países en desarrollo en la década del noventa, apenas fueron significativos en cuanto a la reducción del número de dependencias gubernamentales y de los funcionarios a su servicio (Middlebrook y Zepeda, 2003: 38).

En el caso de los países latinoamericanos, a finales de la década del ochenta la manutención de gobiernos como el peruano, el ecuatoriano y el venezolano rondaba en torno al 35\% del PBI; y el mexicano, el argentino y el brasileño, en torno al 38,5\% del PBI (Mattelart, 2004: 75-77, y Stokes y Hernández, 2002: 92). Lo interesante es que hoy en día, a casi veinte años de los primeros intentos que dibujaron una redefinición de las competencias del Estado, aún pervive en el ideario de muchos políticos latinoamericanos la añoranza por la vuelta a casa, lo que equivale a que se retome la vieja senda del anacrónico populismo y se le dé nuevos bríos a la estéril creencia de que es condición de un Estado fuerte ser grande, sin detenerse un ápice a cuestionarse quién debe sufragar tal situación (Garzón y Salomón, 2006: 63-65).

\section{Conclusiones}

En el sistema capitalista de talante liberal, el Estado desempeña un papel de garante y valedor respecto de: a) la protección de la propiedad privada y la libertad económica a través de la creación de marcos legales, b) la promoción y la protección de los intereses de los individuos y de las empresas privadas, c) el respeto a la legalidad, d) al cumplimiento de las reglas creadas para el fomento de la libre y leal competencia y e) el respeto al equilibrio logrado por consumidores y oferentes sobre los niveles de producción y los precios, entre otros aspectos.

El control que ejerce el Estado sobre los aspectos estratégicos de la economía se evidencia, con frecuencia, a través de una responsabilidad pública cuyos propósitos son: a) el respeto a la legalidad, b) lo que se entienda por equidad social, c) lo que se entienda por ejercicio del libre comercio, d) la defensa de la soberanía nacional y e) el sentimiento de protección a los sectores más desfavorecidos de la población, entre otros.

Mientras que el mercado desempeña su labor de coordinación a través de mecanismos como el precio, el Estado aporta un conjunto de instrumentos que son complementarios al mercado y las empresas; el correcto y diligente funcionamiento de estos instrumentos genera certidumbre y estabilidad en los mercados, mientras su ausencia, su parcialidad, su torpeza o su ineficiencia generan incertidumbre, la cual se traduce en elementos que incrementan el riesgo.

La visión yuxtapuesta entre el Estado y el mercado tiende a evaluar si es posible: a) repartir sin crear, b) repartir más de lo 
que se es capaz de crear, c) repartir menos de lo que se es capaz de crear o d) inhibirse de repartir y confiar en que el mercado se encargará de hacerlo de la mejor manera posible. El problema es que los gobiernos muchas veces olvidan que para crear, antes es preciso lograr la asignación eficiente de los recursos, la preeminencia de una sana competitividad y el incremento de la productividad, lo que en términos sociales se traduce en que todos los individuos que forman la sociedad tengan la posibilidad de mejorar sus condiciones de vida, lo cual no necesariamente implica una lucha por la igualdad.
El género humano forma parte simultáneamente del ámbito de competencia del Estado y del mercado. Por tanto, los cambios en el dominio del Estado (poder) necesariamente afectan a los individuos en la economía, y los cambios en el dominio del mercado (riqueza) necesariamente afectan la asignación y la distribución del poder del Estado. Es un hecho que la configuración de un sistema económico-político eficiente se somete permanentemente a las condiciones que establecen la asignación y la distribución de la riqueza y el poder, así como a los diferentes intereses y valores que persiguen tanto el Estado como el mercado.

\section{Referencias bibliográfícas}

AMATTO, Giulio. 2004. Un analisi sull mito della convergenzia. Milano: Il Mulino.

BOBBIO, Norberto. 1989. Estado, gobierno y sociedad. México: Fondo de Cultura Económica.

BUCHANAN, James y TULLOCK, Gordon. 1962. The Calculus of Consent: Logical Foundations of Constitutional Democracy. Ann Arbor: University of Michigan Press.

1969. Cost and Choice. Chicago: Markham (Midway Reprint, University of Chicago Press, 1976).

- 1975. The Limits of Liberty. Chicago: University of Chicago Press. 1985, Liberty, Market, and State. New York: New York University Press.

1992. Better than Plowing and Other Personal Essays. Chicago: University of Chicago Press.
2004. The Collected Works of James M. Buchanan. Indianapolis: Liberty Fund.

COASE, Ronald. 1963. Contract Acts and Citizens Relations. New York: Routledge.

1999. Selected articles of Ronald Coase. Boston Mass.: Harvard University Press.

CUADRADO ROURA, Juan Ramón (coord.). 2005. Introducción a la política económica, Madrid: Mc Graw Hill.

FUÀ, G. y MATERAZZI, C. 2004. Lo Stato modellico italiano. Roma: La Sapienza.

GARCÍA MAYNES, Eduardo. 1994. Introducción al estudio del Derecho. 48. ${ }^{\mathrm{a}}$ ed. México: Porrúa.

GARZÓN, Pablo y SALOMÓN, Ignacio. 2006. El estado de las políticas públicas en América Latina a debate. Buenos Aires: Universidad de Buenos Aires. 
MATTELART, Xavier. 2004. Quince años de política desregulatoria en América Latina. México: Taller Abierto.

MIDDLEBROOK, Keith y ZEPEDA, Eduardo. 2003. Confronting Development: Assesing Mexico's Economic and Social Challenges. Stanford: Stanford University Press.

\section{PÉREZ-PRENDES Y MUÑOZ DE ARRACO,} José Manuel. 1998. Tratado de historia del Derecho. Madrid: Universidad Complutense de Madrid.

RODRÍGUEZ BRAUN, Carlos. 2005. Diccionario políticamente incorrecto. Madrid: Editorial Lid.

RONCAGLIOLO, Paolo. 2003. Lo Stato, tra lo pubblico e lo privato. Roma: La Pérgola.

SALVON, John y SKA, Gulden. 2002. How do Helsinski's Public Sector Works. Helsinski: University of Helsinski. Working Paper Series N. ${ }^{\circ} 023$.
SANTIBÁÑEZ, Luis y PUIGCERDÁ, Denia. 2005. Análisis empírico de las variables relacionadas con la presión fiscal en los países de la Unión Europea (1995-2003). Madrid: Universidad Autónoma de Madrid, Departamento de Economía. Serie Papeles de Trabajo n. ${ }^{\circ} 34$.

SCHUMPETER, Joseph A. 1942. Capitalism, Socialism and Democracy. Boston: Mass., Harvard University Press.

SMITZ, James y ZUBIZARRETA, Antxon. 2003. El coste de la democracia y su administración. Valencia: Tirant lo Blanch.

STOKES, Edelbert y HERNÁNDEZ, Roberto. 2002. Economy of Mexico. New York: Mason Crest Publishers.

VUSCOVIC, Petra. 1975. Dos años de política económica del gobierno popular. México: Fondo de Cultura Económica. 